\title{
Research on Word-of-mouth Communication Mechanism Based on Characteristics of Source Propagation Nodes
}

\author{
Lei WANG ${ }^{\mathrm{a},{ }^{*}}$, Min $\mathrm{ZHAO}^{\mathrm{b}}$, Rui-Dian $\mathrm{ZHANG}^{\mathrm{c}}$
}

School of Economics and Management, Lanzhou Jiao tong University, Lanzhou 730070, China

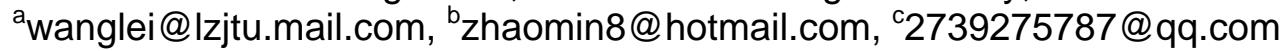

Keywords: Word-of-mouth communication, Source propagation node, Complex network, Activity.

\begin{abstract}
This article used complex networks and other tools to study how word propagation affects different characteristics of source networks in complex networks. First, based on the previous research, we integrated the SIR virus transmission model with various factors that influence the spread of word-of-mouth communication, mainly the word-of-mouth type and node characteristics, and established a word-of-mouth communication; Then, according to the acquired blog data, we constructed a complex network, analyzed the characteristics of the nodes of the network, and selected nodes with different characteristics as source nodes according to the degree and activity of the nodes; Finally, we embedded the built word-of-mouth communication model in the actual blog data to simulate, and measured the effects of different source nodes on the evolutionary trends of susceptible nodes, infected nodes, and immune nodes. The experimental results show that the source nodes characteristics have great effect on word-of-mouth communication.
\end{abstract}

\section{Introduction}

With the continuous development of the Internet, communication methods have undergone great changes. Internet word-of-mouth communication has, replaced past word of mouth. Therefore, we can use the complex network based on user-node and user-to-user relationship as the basic framework to study the mechanism of Internet word-of-mouth communication.

At present, scholars at home and abroad research on word-of-mouth communication based on complex networks, focusing on the influence factors of word-of-mouth communication and their effects. Its content focuses on the influence of word-of-mouth, customer awareness and network structure parameters on the spread of word-of-mouth.

From the point of view of word-of-mouth type, Jin Liyin [1] (2007) studied the direction of Internet word-of-mouth information and the influence of the subjectivity and objectivity of Internet word-of-mouth on the effectiveness of Internet word-of-mouth communication. The results of the study found that the effect of negative word-of-mouth on the consumer's buying decision was significantly higher than positive word-of-mouth.

From the perspective of network structure parameters, Vilpponen et.al [2] (2010) used social network analysis to study the influence of word-of-mouth network structure on word-of-mouth communication effectiveness. The study also found that the centrally located nodes in the network have a great influence and are more conducive to the proliferation and spread of new product information. Li Lin, Sun Jun, and Zhou Zhangjin[3] studied the differences in the heterogeneity of nodes and the topology structure of the network and brought differences in propagation probabilities and propagation ranges.

From the perspective of node characteristics, Yang Yanyang et al.[4] assumed that the customer's dissemination is directly proportional to the customer's awareness. Therefore, customers with higher awareness have higher spread and are more important in the network. Bi Jidong [5] (2009) empirical research based on TAM theory finds that relational intensity is the most important factor influencing consumer purchases for negative word-of-mouth communication, and that professional perception of information recipients negatively affects consumers' purchase intentions. Zhang Yanchao [6] studied the information dissemination of online social networks and found that the greater the degree of the initial propagation node, the faster the information dissemination. 
The word-of-mouth communication model constructed in this paper is a combination of word-of-mouth and node characteristics. In the process of researching the model simulation, it focuses on the impact of the characteristics of the source propagation node on different nodes.

\section{The Establishment of a Model}

\section{Information Dissemination Model}

This article divides the user density into three categories as time changes. Online social networks are often abstracted as complex networks where nodes represent users and information on the network is propagated through the edges between nodes [7]. That is, an infected unknown person can become a communicator with a certain probability and become an immune communicator after a certain period of time [8]. Zhang Haifeng [9] used the framework of virus propagation and the probability of connection between nodes in his research to construct an information dissemination model, the user density s(t) in a susceptible state, the user density $\mathrm{i}(\mathrm{t})$ in an infected state, and the user density $\mathrm{r}(\mathrm{t})$ in an immunized state. Its propagation rules are described as follows:

(1) During each period of time, if the vulnerable node is connected to the infected node, the vulnerable node will be infected with a certain probability $\lambda$ and enter the infection state.

(2) The infected node does not remain in an infected state. At time t, it will be cured with a certain probability $\gamma$ and become an immune node. It will be immune. It will not infect the disease again and it will not infect other nodes.

This model is the change of the density of different nodes in the process of propagation, which describes the whole process of information dissemination.

\section{Word-of-mouth Communication Model}

\section{Model Construction}

In the above model, the connection mode of the node is not considered. In the process of word-of-mouth communication, the connection of nodes is mainly affected by the degree of association of nodes, so the degree of association $p\left(k^{\prime} / k\right)$ is introduced into the model. In addition, considering the influence of word-of-mouth and node characteristics, both of them mainly affect the propagation probability $\lambda$ and the immune probability $\gamma$, thereby affecting the overall communication effect of word-of-mouth. In the context of degrees, the relationship between connection probability and degree is $\theta_{k}(t)=\sum_{k^{\prime}} p\left(k^{\prime} / k\right) i_{k}(t)$.In order to take this factor into account, therefore change Zhang Haifeng's relational model about $\theta_{k}(t)$ in the information dissemination model to the above relationship based on degree correlation. The overall structure of the word-of-mouth communication model constructed in this paper is as follows:

$$
\left\{\begin{array}{l}
\frac{d s_{k}(t)}{d t}=-\lambda k s_{k}(t) \theta_{k}(t)-\gamma s_{k}(t) \\
\frac{d i_{k}(t)}{d t}=\lambda k s_{k}(t) \theta_{k}(t)-i_{k}(t) \\
\frac{d r_{k}(t)}{d t}=i_{k}(t)+\gamma s_{k}(t)
\end{array}\right.
$$

In equation (1), $\mathrm{k}$ indicates the degree of the node. $\theta_{k}(t)$ shows the probability that any given edge of a node of degree $\mathrm{k}$ is connected to an infected node. $s_{k}(t)$ represents the density of susceptible nodes with degree $\mathrm{k}, i_{k}(t)$ represents the density of propagation nodes with degree $\mathrm{k}$, and $r_{k}(t)$ represents the density of immune nodes with degree $\mathrm{k}$. The infection probability $\lambda$ and the immune probability $\gamma$ are constructed as $\lambda(m)=\mu m /(1+\eta m) \gamma(n)=a-b e^{-\mu m}$. The construction of these two formulas will be specifically expanded in the following parameter descriptions. 


\section{The Model Parameter Influence Mechanism is Explained}

In the word-of-mouth communication model constructed in this paper, the main parameters considered are the infection probability $\lambda$ and the immune probability $\gamma$, and the two parameters are mainly based on word-of-mouth and node characteristics. Usually, higher-level and more active nodes will make word-of-mouth communication easier. Normally, negative word of mouth has a stronger effect on communication. People are more willing to spread and have greater impact on people. Based on these two factors, these two mechanisms are elaborated below.

The following two mechanisms are constructed as the following equations:

(1) Based on the above explanation, during the entire propagation process, the number of neighboring nodes has a large influence on the spread, and thus the formula for the probability of infection is $\lambda(m)=d \times \mu m /(1+\eta m) . \lambda$ is the probability that a susceptible node becomes an infected node, $\mu$ refers to customer awareness, $\eta$ refers to the level of word-of-mouth, $\mathrm{m}$ is the number of neighboring nodes in a susceptible state, and d refers to the activity of the node;

(2) The customer awareness and the immune probability are positively related, but its impact on the immune probability is not infinitely increased, so the exponential function formula is used. That is $\gamma(n)=a-b \bar{e}^{-m} \cdot \gamma$ refers to the probability of an infected node becoming an immune node, a represents the maximum awareness, $b$ refers to the adjustable coefficient, $\mu$ refers to the customer's awareness, and n refers to the number of neighboring nodes in the infected state;

The above two equations mainly describe the influence of word-of-mouth type and node characteristics on propagation probability and immune probability.

\section{Model Simulation Analysis}

This article is using thousands of data on micro blogging to research online word-of-mouth communication. Simulation research mainly selects different nodes as source propagation nodes through the different characteristics of network nodes, and examines the impact of different source nodes on susceptible nodes, infected nodes, and immune nodes, and finally concludes that the characteristics of source propagation nodes affect the entire propagation behavior.

\section{Data Acquisition and Analysis}

This article is based on the popular platform - Sina Weibo's open port API to get the relevant data of the user relationship, mainly used to build the corresponding user relationship network. Therefore, the article selects 1224 nodes, which forms 20,316 relationship lines, and uses Pajek software to analyze the complex network diagram as follows in Fig.1:

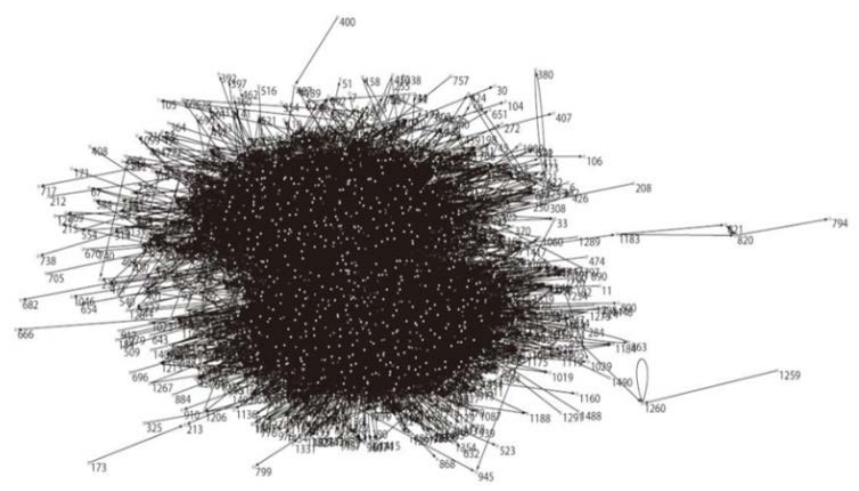

Fig.1 The complex network diagram

The network has a degree of aggregation of 0.1785 and an average degree of 31.0817. It can be seen that the degree of aggregation of the network is not particularly high, and each node is connected to 31 nodes on average. This ensures the extent and speed of information transmission in 
the network to some extent.

\section{Source Propagation Node Selection}

The paper combines the degree and activity of the node. First analyze the degree distribution of the entire network, and then select the corresponding node in the node with the highest degree, the node with the least degree, and the node with the average degree respectively. At the same time, the activity of related nodes is obtained through tracking, and the nodes with high activity and low activity among the nodes with similar degrees are selected as the source propagation nodes, and then it is examined how different source propagation nodes influence the changes of the three types of nodes.

The activeness of this paper is mainly the percentage of the landing time of the node within one month, the degree and activity of the integrated nodes. In order to facilitate the study, the following six nodes are selected. The following table shows the degree and activity of each node.

Table 1. Characteristics of Source Propagation Nodes

\begin{tabular}{|l|l|l|}
\hline Node number & degree & Activity \\
\hline 9 & 1 & 0.203702 \\
\hline 46 & 1 & 0.807116 \\
\hline 60 & 118 & 0.344839 \\
\hline 801 & 123 & 0.859433 \\
\hline 674 & 301 & 0.210154 \\
\hline 127 & 351 & 0.835131 \\
\hline
\end{tabular}

From Table 1, it can be seen that in the selection of source propagation nodes, the two dimensions are mainly from degree (high, medium, low) and activity (high, low). By comparing the influence of different source propagation nodes on the evolution trends of the three types of nodes, the influence of the characteristics of source propagation nodes on word-of-mouth communication behavior is investigated.

\section{Source Propagation Node's Activity and Degree on Characteristic Nodes}

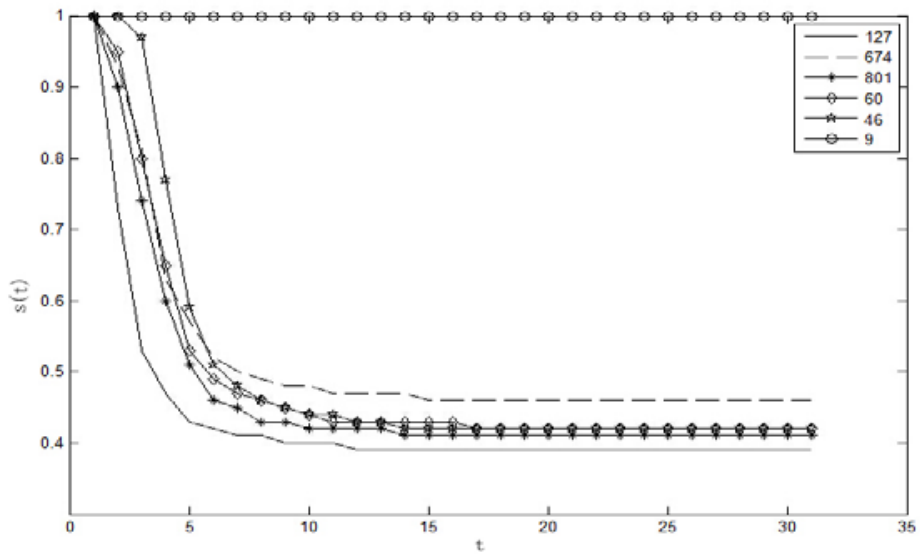

Fig. 2 Trends of susceptible nodes with different source propagation nodes

Fig.2 describes the impact of different source propagation nodes on changes in susceptible nodes. Through the above comparison observations can be found: by comparing node 801 and node 674, it is possible to cross-comparison the influence of nodes with different degrees and activity on the susceptible nodes, and the node 801 that is at a medium level and whose activity level is higher than the node 674 that is less active than the altitude is expected to decrease fast. To illustrate, relatively speaking, the degree of activity is more significant than the degree of node for susceptible nodes, that is, its influence on word of mouth communication is more obvious. 


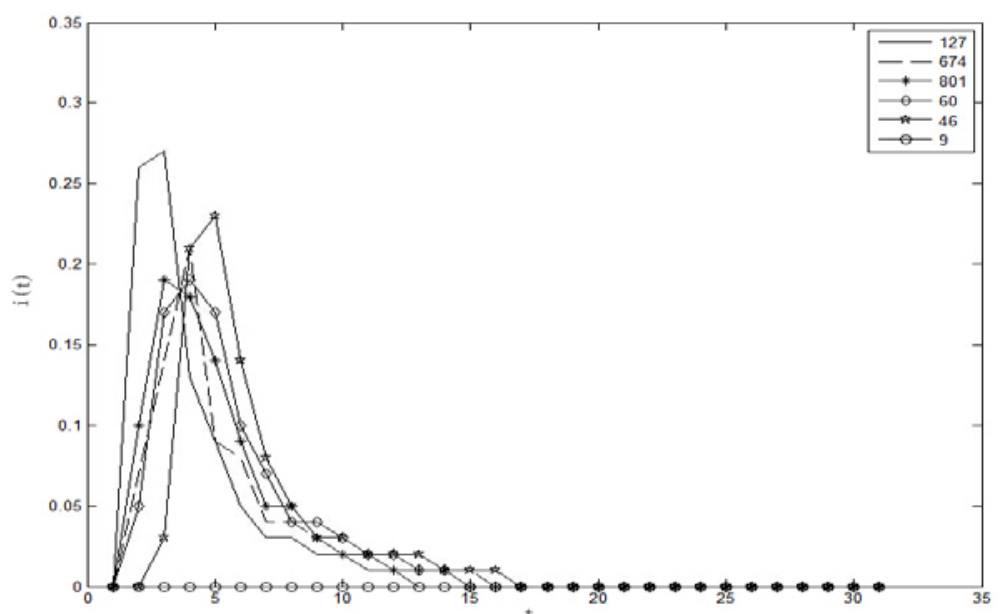

Fig. 3 Trends of infected nodes with different source propagation nodes

Fig.3 illustrates the impact of different source propagation nodes on the change of infected nodes. Through the above comparison observation can be found: in the low activity degree, the source propagation node with a medium level promotes the fastest propagation speed, while at a high activity level, the higher the node speed is, the faster the node promotes. Then, by comparing node 801 and node 674, it is possible to cross-compare the influence of the source propagation node with different degrees and activity on the infected node. To illustrate, relatively speaking, the degree of activity of the source propagation node is more significant than that of the node.

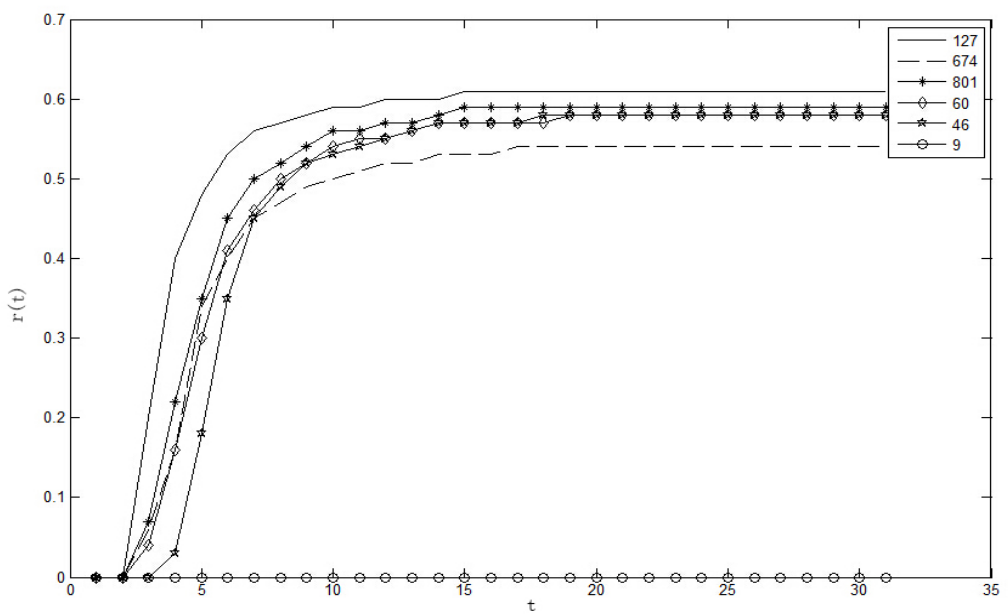

Fig. 4 Trend of immune nodes with different source propagation nodes

Fig.4 illustrates the impact of different source propagation nodes on the change of immune nodes. From the figure above, we can see that when the node 9 with the lowest customer awareness and the lowest activity is used as the source propagation node, the immune node is always at zero, indicating that it is difficult to enter the immune state at this time; When the node 127 with the highest customer awareness and highest activity is the source propagation node, it is in the opposite state. The speed of pushing the node into the immune state is the fastest among all nodes, and the enterprise should pay attention to these nodes in time to prevent users from entering the immune state prematurely.

\section{Marketing Advice}

For enterprises, when examining the impact of source propagation nodes on word-of-mouth communication, attention should be paid not only to nodes with higher node degrees, but also to 
those nodes that are less high but more active. In the word-of-mouth marketing, enterprises must first take the more active star users as the first choice for marketing, focusing on turning these users into corporate and product spokespersons; Secondly, we must develop the micro blogging reds in social networks for those who are more active users, and turn their activities into a way for corporate propaganda; Finally, through some social channels, efforts are made to increase the enthusiasm of the users involved in the company, and to truly achieve "word of mouth" to form a reputation for the company's reputation.

\section{Acknowledgement}

This article is supported by National Natural Science Foundation of China under grant No. 71461017.

\section{References}

[1] Liyin Jin. The Impact of Internet Word-of-Mouth Information on Consumers' Purchase Decision: An Experimental Study, Economic Management, 2007, 29(22):36-42(In Chinese).

[2] Vilpponen et.al. Electronic Word of Mouth in Online Environments: Exploring Referral Network Structure and Adoption Behavior, Journal of Interactive Advertising, 2006,6(2): 63-77.

[3] Lin Li, Junhua Sun, Zhang Zhou. Service quality word-of-mouth communication based on complex social networks ,Systems Engineering, 2009, 6(In Chinese).

[4] Yanyang Yang et al. Analysis of the stability of complex customer networks, Mathematics in Practice and Theory, 2013, 43(23): 113(In Chinese).

[5] Jidong Bi. Empirical Study on the Impact of Internet Word of Mouth on Consumers' Purchase Intention, Journal of Information, 2009,28(11): 46-51(In Chinese).

[6] Yanchao Zhang, Yun Liu, Haifeng Zhang, et al. Information propagation model based on online social networks, Acta Phys. Sin, 2011, 60(5): 35(In Chinese).

[7]Weigang L, Sandes E F O, Zheng J. Querying Dynamic Communities in Online Social Networks, Journal of Zhejiang University SCIENCE C, 2014, 15(2): 81-90.

[8] Zhao X, Wang J. Dynamical Model About Rumor Spreading with Medium, Discrete Dynamics in Nature and Society, 2013(10): 58.

[9] Haifeng Zhang. Dynamic Behavior of SIR Epidemic Model with Immunity on Complex Networks, Journal of Shanghai University, 2007, 13(2):190-191(In Chinese). 